\title{
Development of remote meter reading system based on GSM MODEM
}

\author{
Yin-Jing Guo, Xiao-Li Yi, Lu Zhang, Chang-You Ji, Zhen-Zhen Liu and Shi-Peng Zhang \\ College of Electronic Communication and Physics, Shandong University of Science and \\ Technology, Qingdao, 266590, Shandong, China \\ Email:gyjlwh@163.com \\ www.sdust.edu
}

\begin{abstract}
In this paper, we develop electricity meters auto-transcription system by using C\# and GSM MODEM based on C/S model. Compared with the original meter reading system, the performance of this system has been greatly improved. Under the premise of remote data transmission functions such as residential account management, automatic metering and payment, using detailed query printing, the system achieved the SMS function. The system was completed by adopting SQL data management system and ADO.NET technology, while AT commands control of SMS modem was achieved by directly calling API functions. Application results show that the system can improve the mobility of the residential manager's work and the convenience of the residential users who need to use the electricity payment query.
\end{abstract}

Keywords: ADO.NET; Remote Meter-reading; SMS Function.

\section{Introduction}

Remote meter reading system is widely applied, and it makes up for the shortcomings of ordinary manual meter reading, with many advantages such as saving of human resources, helping management to grasp the exact data, improving office efficiency[1,2] .

Systems developed using $\mathrm{C}$ \# have good application interface, safe and reliable function and good usability[3].On the basis of meter remote meter reading system ,we has developed a remote meter reading system which has SMS functionality through using of C \# and GSM MODEM. The system have the basic transmission functions for example the management of residential district account, automatic meter reading, payment, check printing, sending message. System establishes efficient communication channels between managers and users. Managers can send text messages to the user in time to remind users of not enough power. 


\section{System Design}

\subsection{System functional design}

Referring to more convenient management of cell electric meters as the principle, based on GSM MODEM, such system want to use .NET technology to build an intelligent meter reading payment platform that using community management department to be data managing centrality and can achieve the function of SmsGroupSends. System can provide visualization services of the remote reading data for the community management department, and numerous and complex meter data can be presented to community managers in a simple and orderly manner. The function of the system is shown in Fig. 1.

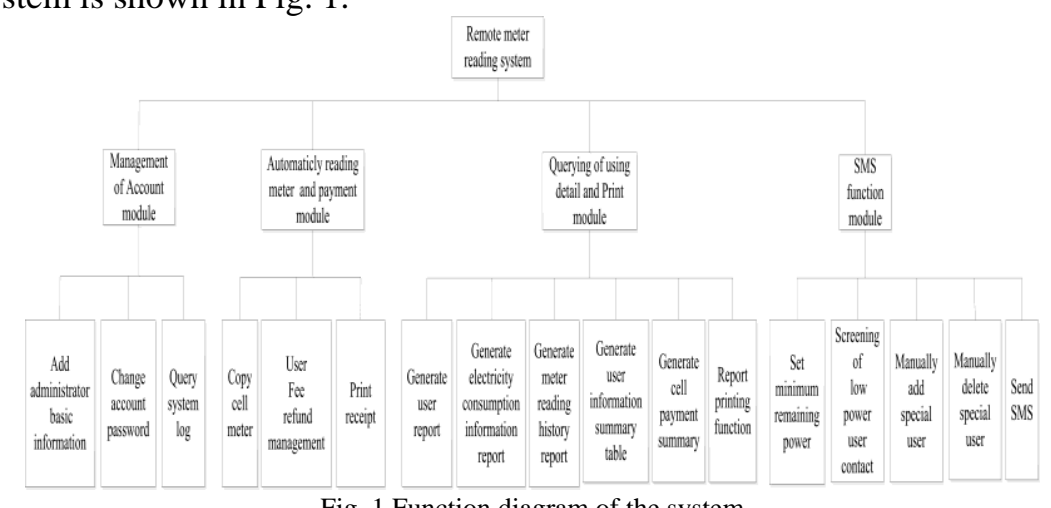

\subsection{GSM MODEM hardware connection}

GSM MODEM using wirelessly implement messaging functionality. It contains text messages sending module and simplify communication interface that supports to send and receive text messages to users [4].In this system, using USB to make SMS modem connected to the computer, through a set of AT commands, GSM MODEM can achieve groups SMS function in accordance with the selection criteria set by the user [5]. SMS modem connection is shown in Fig. 2. 


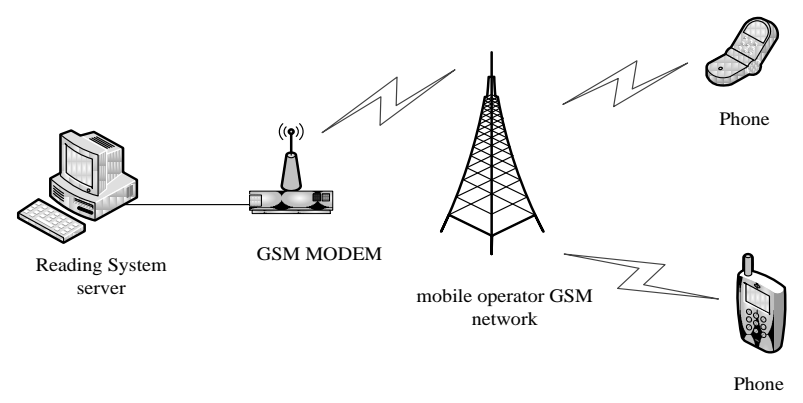

Fig. 2 GSM MODEM connection diagram

\section{The Key Technology of System Development}

This article based on Visual Studio 2010 and C/S mode, use C\# and Microsoft SQL Server 2008 to complete development of the system.

\subsection{Database design and connection}

We integrate data information which needs to be stored and analyzed in system. The data which have similar properties will be placed in the same data table. The 11 static tables and the information contained in the main field are listed in Tab. 1.

Tab. 1 Mainly static data table in system

\begin{tabular}{|c|c|}
\hline Table Name & Information contained in the field \\
\hline users & the basic user information \\
\hline Area & $\begin{array}{l}\text { community address, electricity price, lowest power and other auxiliary } \\
\text { information }\end{array}$ \\
\hline dabh & community and sub-table information \\
\hline client & information of system administrator \\
\hline $\mathrm{rcb}$ & Day meter reading and monthly reading information \\
\hline jiaofei & Remaining power, unit price, total electricity payment information \\
\hline duanxin & Users' information need to send text messages \\
\hline SmsSend & Message content, the transmission time, the successful identification, etc. \\
\hline $\begin{array}{l}\text { SmsSendTe } \\
\mathrm{mp}\end{array}$ & SMS History \\
\hline sys-log & operation recording of system \\
\hline parameter & Normal system operating parameters \\
\hline
\end{tabular}

ADO.NET is used to connect database [6,7], the connection string as follows: Public static string connectionString = "Data Source=localhost; Initial Catalog=chaobiaoDB; User ID=Login account; Password=Password". 


\subsection{GSM MODEM application mode}

GSM MODEM has several different application modes. AT commands can be used directly, or used secondary development package of AT commands integrated [5]. In such system, secondary development package is used. When developing system, we only need to directly call API functions what are encapsulated in sms.dll. API functions are shown in Tab. 2.

Tab. 2 API function with SMS modem

\begin{tabular}{cc}
\hline API subprogram & Function \\
\hline Sms_Connection() & SMS modem connection \\
Sms_Disconnection() & SMS modem Disconnect \\
Sms_Send() & send messages \\
Sms_Receive() & receive messages \\
Sms_Delete() & delete messages \\
Sms_AutoFlag() & Messages automatically receive identification \\
Sms_NewFlag() & news logo \\
\hline
\end{tabular}

\subsection{SMS function realization}

Because in the development process to perform various operations on GSM MODEM, such as getting communications port and baud rate, so the system defines a GSM class which include a variety of methods, and quotes the namespace using System. Runtime. InteropServices to invoke sms. dll dynamic link library.

\subsubsection{GSM MODEM connecting}

When sending messages, community managers need to select GSM MODEM port in the system interface. If SMS modem can be normal connected, the interface will show "GSM MODEM connection is successful!". If the GSM MODEM can't be properly connected, interface is displayed "connection failed, please re-confirm the port number." After successful connection, managers can use its text-messaging capabilities.

\subsubsection{Sending messages}

In order to send messages, the system uses the SMS Send function which is belong to sms.dll. The manager of community usually filtrate the target number through selecting community and the minimum electric quantity, to filtrate suitable number from database accord to the manager's choice and show in dataGridView1 sheet. Besides, the manager of community can add 
additional number which the databases have not or delete numbers in sheet accord to the actual demand. Firstly, the system judge the number which stored in the sheet of dataGridView1 and the length of messages what be imported by managers of SendSms_Text whether meet the requirements, then send messages one by one according the numbers in the list. The subfield of dataGridView1 can be used to display whether sent successfully. After send messages, the basic information like contents and time and others be stored in data sheet uniformly.

\subsubsection{The application example of message function}

Add a testing community to test the capabilities of SMS model. When setting minimum power, message can be send to users whose power falls below the value. Historical query interface shown in Figure 3.

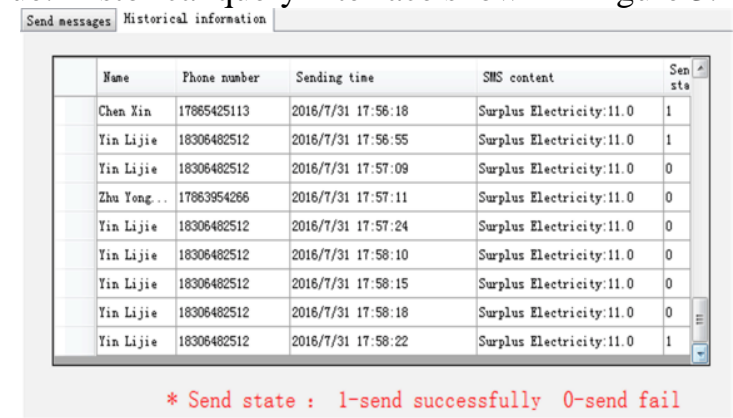

Fig. 3 History information query

The button of adding manually provides the function of adding special users' information for manager flexibly. As shown in figure 4.

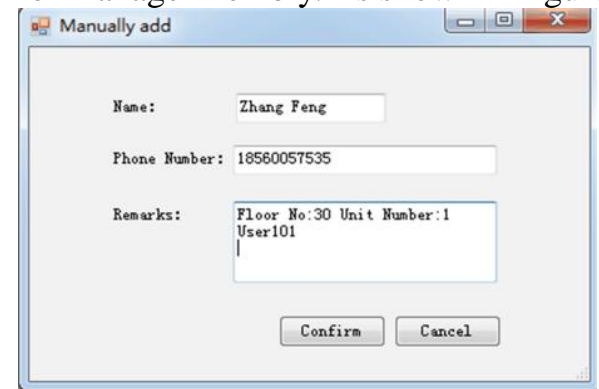

Fig. 4 Adding special users manually

\section{Conclusion}

The remote meter reading system based on GSM MODEM adds a message 
function besides the functions of account management, automatic meter reading and pay cost, using subsidiary query print that can help the managers and users to communicate with messages in time. The application results indicate that the capability of older version has been greatly improved, the mobility of managers' work and economic benefit also increased obviously. At the same time, users can get some information of electricity consumption situation and payment, not have to go to relevant departments of power supply bureau to check it. It can save time and energy.

But the system still needs to improve and perfect in the following aspects.

(1)When the system working, you must restart software after plug GSM MODEM again, the stability of information transmission is restricted;

(2)When the system transfer large amount of information, information is not delivered to users in time, the timeliness of information transmission is restricted.

\section{Acknowledgments}

I would like to express my gratitude to all those who helped me during the writing of this thesis. And this work is supported by grant 61471224 of the China National Science Foundation and grant BS2013DX016 of Shandong Outstanding Young Scientist Award Foundation.

\section{References}

1. Ma Zi Yan, Design of remote meter reading system based on SMS, Microcomputer Information, 2006, 22(7):165-166.(In Chinese).

2. Liu Yang, Ren Gong Chang, Miao Xin Qiang, Li Zhong Yan, Design and implementation of GPRS-based remote meter reading systems, Machinery Design and Manufacture,2011,3:248-250.(In Chinese).

3. Wang Hai Yan, The development of three layer architecture of database application system under C\#.NET, The Computer Technology and Development, 2012, 06:78-81.(In Chinese).

4. Liu Fang, Design and implementation of short message sending system based on GSM modem, Computer Knowledge and Technology,2013,19(31):7002-7005.(In Chinese).

5. Li Shu Kun, The design and development of college course aided system based on GSM MODEM, Computer Applications and Software, 2012, 04:203-205+208.(In Chinese).

6. Guckenheimer S, Perez J J, Software Engineering with Microsoft Visual 
Studio Team System (Microsoft. NET Development Series). AddisonWesley Professional, 2006.

7. Cao Jian, Lu Hong Yan, Based on the analysis of three layer architecture of database application system development under C\#.NET, Information Systems Engineering, 2013, 05:43.(In Chinese). 